\title{
Kinetics of N, N-Dimethyaniline-Benzenesulphonylchloride Charge-Transfer Complex Initiated Cyclopolymerization of Divinyl Monomer
}

\author{
Krishnaveni Nachimuthu (Corresponding author) \\ Department of Chemistry, Velalar College of Engineering and Technology \\ Thindal, Erode-638012, Tamilnadu, India \\ E-mail: venisundar_erd@rediffmail.com \\ Umayavalli Manickam \& Sivakumar Genesan \\ PG \& Research Department of chemistry \\ Arulmigu Palani Andavar College of Arts \& Culture \\ Palani-624601, Tamilnadu, India \\ E-mail: umayavalli@gmail.com
}

Received: April 8, 2011 Accepted: April 30, 2011 doi:10.5539/ijc.v3n3p93

\begin{abstract}
Polymerization of non-conjugated divinyl monomer N,N'-methylenebisacrylamide was studied using a novel charge-transfer complex of N,N-dimethylaniline-benzenesulphonyl chloride in a homogeneous gel-free state, under varying conditions of concentration and temperature. The reactions were carried out under various concentrations at a constant temperature of $52{ }^{\circ} \mathrm{C}$ in acetonitrile media. The dependence of the rate of polymerization on various experimental conditions, such as different concentrations of monomer N,N'-methylenebisacrylamide, donor N,N-dimethylaniline, acceptor benzenesulphonyl chloride, and temperature were studied. The order with respect to monomer, donor, and acceptor were found to be $1,0.5$, and 0.5 respectively. The kinetic results were interpreted by a suitable mechanistic sequence. Absence of gelation is attributed to the formation of a cyclic polymer. Jobs method of continuous variation studies shows 1:1 complexformation.
\end{abstract}

Keywords: Cyclopolymerization, Non-conjugated divinyl monomer, N,N-dimethylaniline-benzenesulphonyl chloride, Charge-transfer complex

\section{Introduction}

Modern technology development needs reliable high-performance polymers for advanced applications. Bulk synthesis of polymers involves various kinetic factors. Kinetic studies play a major role in the industrial synthesis of many high-performance polymers (Michael A. Meador., 2003). N,N'-methylenebisacrylamide (MBA), which is a symmetrical non-conjugated diolefin having a double bond in the terminal position, is a well-known cross-linking monomer used in the preparation of hydrogels, which are studied for the development of selfregulated insulin delivery systems (Satis, et al., 2006) and as a potent anticancer drug. The formation of insoluble gel was due to the three-dimensional growth of the polymer. This type of monomer can also polymerize without cross-linking to give soluble gel-free polymer (Gopalan, et al., 1982; Subbaratnam, et al., 1986; Ratnasabapathy, et al., 1988). Butler and his co-workers reported that a monomer containing three or four cyclic groups leads to the formation of insoluble polymers. A chain growth mechanism was proposed involving an alternation of intra and intermolecular steps, which for the first time was found to lead to cyclopolymerization (Butler, et al., 1957). The cyclopolymerization of $\mathrm{N}, \mathrm{N}^{\prime}$-methylenebisacrylamide initiated by an eosin-ascorbic acid system is reported. The rate of polymerization is proportional to [MBA] and [Ascorbic acid] (Parthasarathy, et al., 2004). The cyclopolymerization of non-conjugated divinyl monomer N,N'-methylenebisacrylamide with a redox pair of $\mathrm{Mn}$ (VII)-Thiol in a homogeneous gel-free state in the authors' laboratory is also reported here (Umayavalli et al., 1996). Park et al. is reported the selective cyclopolymerization of alpha, omega-dienes (Park, et al., 2006). 
Recently, the Pd (II)-dimine catalysts initiate cyclopolymerization of isopropylidene diallylmalonate(1) to produce the polymer contaning trans-1,2-disubstitued cyclopentane groups has been reported (Park, et al., 2010).

A charge-transfer complex has not been studied previously as an initiator for cyclopolymerization. The present investigation of cyclopolymerization of non-conjugated divinyl monomer initiated by $\mathrm{N}, \mathrm{N}$-dimethylaniline-benzene sulphonyl chloride charge-transfer complex indicates that N,N-dimethylaniline (DMA) functions as a lone pair n-donor of the increvalent type. This is proved by its ineffectiveness in initiating polymerization in the presence of hydrochloric acid. It is a well known n-donor. It also behaves as a $\pi$-acceptor when it interacts with another suitable $\pi$-acceptor (Kothandaraman, et al., 1986a; Kothandaraman, et al., 1984b). The reasons for choosing benzenesulphonyl (Bscl) chloride as an acceptor were its simplicity and its structure which is analogous with that of O-toluene sulphonyl chloride.

Studies of charge-transfer complexes involving aromatic sulphonyl chloride are relatively rare. The charge-transfer interaction of DMA with O-toluene sulphonyl chloride has been investigated, and this complex-initiated vinyl polymerization of methyl methacrylate and acrylamide in acetonitrile medium has been presented as a radical mechanism. The initiating radical is formed by thermal decomposition of the complex. It was found to be first order to [monomer] and of order 0.5 to [DMA] and [OTscl] (Kothandaraman, et al., 1984c).

\section{Methods and Materials}

The reagents used were purified using standard procedures. The kinetics of polymerization was studied by batch process. The polymerization experiment was carried out as described below.

A solution containing the required amount of monomer, acceptor was taken in the reaction vessel and made up to the fixed volume $(20 \mathrm{~mL})$ with acetonitrile. The solution was then deaerated for 20 minutes with purified nitrogen as to ensure inert atmosphere as well as through stirring. The tube was thermostated at the appropriate temperature in the thermostat. At the end of 20 minutes, required amount of donor DMA solution was added into the polymer tube through a micro funnel, which was subsequently rinsed with $2 \mathrm{mLof}$ acetonitrile. The passage of nitrogen was continued for 5 more minutes. The inlet and outlet tube were closed to maintain the nitrogen atmosphere till the end of a fixed reaction time. Air was blown to arrest the reaction. The blank test was also conducted under the same experimental condition. The rate of monomer disappearance, $\mathrm{R}_{\mathrm{p}}$ was determined by bromometry.

\section{Results and Discussion}

In this study, under the specified experimental conditions used, neither DMA alone nor Bscl alone could initiate effective polymerization. No initiation of polymerization was observed in aerated conditions or in the presence of hydroquinone, all of which revealed the free-radical nature of the polymerization reaction. No induction period was observed, and light did not alter the reaction kinetics, as was proved by obtaining the same kinetic results in the presence and the absence of light. Nevertheless, all experiments were carried out in a reaction vessel covered with black cloth. The role played by acetonitrile in charge-transfer complex formation must be minimal, as indicated by the fact that there was no shift in the DMA absorption band in acetonitrile and that no polymerization occurred in the presence of dimethylaniline alone in acetonitrile medium. This is similar to the CT complex formation and thermal decomposition behavior of a DMA-OTscl system (Kothandaraman, et al., 1984c).

Absorption measurements for the DMA-Bscl mixture in acetonitrile are presented, and the UV spectra of DMA-Bscl are reported (Figure 1). When the concentration of DMA was increased, the optical density of the CT bands increased also, revealing that these bands could be directly correlated to the donor action of the DMA. Increase in the acceptor concentration also resulted in an increase in the absorption of the CT band.

A series of mixtures of the donor and the acceptor in different ratios was prepared by keeping the total concentration constant, and absorption measurements were made for each solution mixture at $\lambda_{\max }$. If components of the mixture were found to have an absorption maximum, this value was subtracted from the observed absorption value. However, the components of the CT complex studied here were chosen to have negligible individual absorption at the absorption wavelengths exhibited by the CT complex. The optical density was plotted against mole fraction, confirming the formation of a 1:1 complex (Table 1; Figure 2). The complex then underwent thermal decomposition, yielding primary radicals. The polymerization process through the intermediate formation of a charge-transfer complex was indicated by the identification of a charge transfer band at $336 \mathrm{~nm}$. Taking all these factors into consideration, the formation of a CT complex between DMA and benzene sulfonyl chloride is shown in Figure 3.

\subsection{Evidence for cyclopolymerization}

The polymer solution formed is found to have no unsaturation which confirms the involvement of both the double bonds in the propagation step, otherwise known as cyclopolymerization. The IR and NMR spectra of the polymer 
formed which gave the conclusive evidence for the seven membered cyclized ring units in polymer (Hatada, et al., 1986; Ohya, et al., 1983) as detailed below.

The IR spectral details of the polymer and monomer are given in Figure 4. The absorption at $1700 \mathrm{~cm}^{-1}$ due to carbonyl function is not affected in the polymer and in the monomer, MBA. However the absence of $-\mathrm{CH}=\mathrm{CH}_{2}$ stretching frequency at $1630 \mathrm{~cm}^{-1}$ in the polymer which well pronounced in the monomer is of significance. This observation clearly indicates the cyclization propagation sequence (Figure 7 step III or IV) and not through intermolecular addition propagation as observed with monovinyl monomers (Figure 7 step II), in which case the pendant double bonds could be detected. It is also noted that the polymers formed do not consume bromine by addition as decided by bromometric estimations.

The results of NMR analysis of the polymer also support the cyclization propagation (step III or IV) rather than the vinyl propagation (step II) as proved by the absence of any signal at $6.15 \delta$ due to the pendant $\mathrm{CH}_{2}=\mathrm{CH}$ - in the polymer (Figure 5). In the cyclopolymerization of acryclic anhydrides (Corfield, et al., 1972; Hatada, et al., 1986: Ohya, et al., 1983), the difference in the chemical shift for methylenic protons in the six membered rings and that for the two intermittent methylenic protons in the five membered rings were differentiated, recorded and ratio of $\mathrm{HH} / \mathrm{HT}$ intramolecular cyclization was also calculated.

On this basis, similar observation could be expected in cyclopolymerization of MBA also. The observed NMR spectra of MBA polymer is shown in Figure 5. For comparison the NMR spectra of polymethacrylate and polymethylmethacrylate are also indicated (as model compounds) in Figure 6, where total HT addition involving six membered rings and 30\% HH cyclization were reported (Corfield, et al., 1972). By analysis of these spectral data it is clear that the MBA undergoes a total cyclopolymerization and it could be only seven membered rings with two intermittent methylenic groups (Figure7, Step III) constituting the polymer and this conclusion supports our earlier prediction of this type of cyclic units.

\subsection{Effect of monomer concentrations on charge-transfer complexes}

The rate of cyclopolymerization at various monomer concentrations with constant [DMA] and [Bscl] was studied. It was found that the rate constant was dependent on the first power of [MBA]. The $\log R_{p}$ vs. $\log$ [MBA] plot was a straight line with a slope of one. The $R_{p}$ vs. [MBA] plot passed through the origin. The slope of this plot permitted the calculation of the complex rate parameter, $\mathrm{k}_{\mathrm{p}} \mathrm{k}_{\mathrm{t}}^{-0.5}\left(2 \mathrm{k}_{\mathrm{d}} \mathrm{K}\right)^{0.5}$. Using $\mathrm{K}$ and $\mathrm{k}_{\mathrm{d}}$ values taken from the literature, (Kothandaraman, et al., 1986a; Kothandaraman, et al., 1984b; Kothandaraman, et al., 1984c). It is possible to obtain $\mathrm{k}_{\mathrm{p}} \mathrm{k}_{\mathrm{t}}^{-0.5}$ in all three monomer variation studies.

Under constant concentrations of monomer and Bscl, the DMA concentration was varied from $6.7 \mathrm{mmol} \cdot \mathrm{L}^{-1}$ to 33.3 $\mathrm{mmol} \cdot \mathrm{L}^{-1}$. The $\log R_{p}$ vs. $\log$ [DMA] plot gave a straight line with a slope of 0.5 , and the $R_{p}$ vs.[DMA] ${ }^{0.5}$ plot was a straight line passing through the origin. The slope obtained from the latter plot permittedthe recalculation of $\mathrm{k}_{\mathrm{p}} \mathrm{k}_{\mathrm{t}}^{-0.5}$ using the value obtained from the monomer variation experiments. To study the effect of Bscl concentration on the rate of polymerization at constant [MBA] and constant [DMA], Bscl concentration was varied from $4.5 \times 10^{-2}$ $\mathrm{mol} \cdot \mathrm{L}^{-1}$ to $36.4 \mathrm{~mol} \cdot \mathrm{L}^{-1}$. It was observed that the rate of polymerization varied with $[\mathrm{Bscl}]^{0.5}$. The $R_{p} \mathrm{vs}$. [Bscl] ${ }^{0.5}$ plot passed through the origin. From these plots, it was possible to calculate $\mathrm{k}_{\mathrm{p}} \mathrm{k}_{\mathrm{t}}^{-0.5}$, obtaining a value in agreement with that calculated from [monomer] variation and [DMA] variation (Table 2; Figures 8 \& 9).

The value of the overall rate constant, $\mathrm{k}_{\mathrm{p}} \mathrm{k}_{\mathrm{t}}^{-0.5}\left(2 \mathrm{k}_{\mathrm{d}} \mathrm{K}\right)^{0.5}$, was obtained from plots of the rate of polymerization versus monomer concentration and from plots of the rate of polymerization versus the square root of the concentration of the initiators; the value obtained was $1.5 \times 10^{-2}$.

To accommodate the observed results, the following reaction mechanism is proposed:

$$
\begin{gathered}
\mathrm{DMA}+\mathrm{Bscl} \underset{\mathrm{C} \stackrel{K}{\longrightarrow} \mathrm{k} \text { C complex }}{\longrightarrow} \mathrm{R}^{\bullet}
\end{gathered}
$$

where $\mathrm{R}^{\bullet}$ is the initiating free radical.

$$
\begin{gathered}
\text { MBA }+\mathrm{R}^{\bullet} \stackrel{k_{i}}{\longrightarrow} \mathrm{M}_{1}^{\bullet} \\
\mathrm{M}_{1} \stackrel{k_{c}}{\longrightarrow} \mathrm{M}_{1}^{\bullet}(\text { intramolecularly cyclized radical) } \\
\mathrm{M}_{2}^{\bullet}+\mathrm{M} \stackrel{k_{p}}{\longrightarrow} \text { (intermolecular cyclization) }
\end{gathered}
$$

In the termination step, a solvent may also be involved, either by primary radical deactivation or by reinitiation.

$$
\mathrm{M}_{\mathrm{n}}^{\bullet}+\mathrm{M}_{\mathrm{n}}^{\bullet} \stackrel{k_{c}}{\longrightarrow} \text { polymer }
$$




$$
\begin{aligned}
& \left.\begin{array}{r}
\mathrm{R}^{\bullet}+\mathrm{DMA} \underset{\mathrm{k}_{0}}{\stackrel{k_{0}^{\prime}}{\longrightarrow}} \text { products } \\
\mathrm{R}^{\bullet}+\mathrm{Bscl} \underset{k_{0}}{\longrightarrow} \text { products }
\end{array}\right\} \text { Primary radical deactivation } \\
& \mathrm{M}^{\bullet}+\mathrm{S} \stackrel{k_{t r, s}}{\longrightarrow} \text { polymer }+\mathrm{S}^{\bullet} \text { (chain transfer to solvent) } \\
& \mathrm{S}^{\bullet}+\mathrm{MBA} \stackrel{k_{i}^{\prime}}{\longrightarrow} \mathrm{SM}^{\bullet} \text { (reinitiation) }
\end{aligned}
$$

All the types of initiation, propagation, and termination steps were considered, and expressions were derived for the rate of polymerization.

$$
\mathrm{R}_{\mathrm{p}}=\mathrm{k}_{\mathrm{p}} \frac{\left(\mathrm{k}_{\mathrm{i}} \mathrm{k}_{\mathrm{t}}\right)^{0.5}\left(2 \mathrm{k}_{\mathrm{d}}\right)^{0.5}[\mathrm{M}]^{1.5}[\mathrm{C}]^{0.5}}{\mathrm{k}_{\mathrm{i}}[\mathrm{M}]+\mathrm{k}_{\mathrm{o}}[\mathrm{D}]+\mathrm{k}_{\mathrm{o}}^{\prime}[\mathrm{A}]^{0.5}}
$$

The equilibrium constant is

$$
\mathrm{K}=\frac{[\mathrm{C}]}{[\mathrm{D}]_{\mathrm{eq}}[\mathrm{A}]_{\mathrm{eq}}}
$$

Where $[\mathrm{D}]_{\mathrm{eq}}$ and $[\mathrm{A}]_{\mathrm{eq}}$ represent the equilibrium concentration of the donor and acceptor respectively.

$$
\begin{aligned}
& {[\mathrm{D}]_{\mathrm{eq}}=\frac{[\mathrm{D}]_{\mathrm{T}}}{1+\mathrm{K}[\mathrm{A}]_{\mathrm{eq}}}} \\
& {[\mathrm{A}]_{\mathrm{eq}}=\frac{[\mathrm{A}]_{\mathrm{T}}}{1+\mathrm{K}[\mathrm{D}]_{\mathrm{eq}}}}
\end{aligned}
$$

Where $[D]_{\mathrm{T}}$ and $[A]_{\mathrm{T}}$ are the initial concentration of the donor and acceptor respectively.

$[D]$ and $[A]$ are the initial concentrations of the donor and acceptor respectively. Under experimental conditions, $1 \gg[\mathrm{A}]_{\mathrm{eq}}$ and $[\mathrm{D}]_{\mathrm{eq}}, \mathrm{C}=\mathrm{K}[\mathrm{D}]_{\mathrm{T}}[\mathrm{A}]_{\mathrm{T}}$.

$$
\mathrm{R}_{\mathrm{p}}=\mathrm{k}_{\mathrm{p}} \frac{\left(\mathrm{k}_{\mathrm{i}} \mathrm{k}_{\mathrm{t}}\right)^{0.5}\left(2 \mathrm{k}_{\mathrm{d}}\right)^{0.5}[\mathrm{M}]^{1.5}[\mathrm{D}]^{0.5}[\mathrm{~A}]^{0.5}}{\mathrm{k}_{\mathrm{i}}[\mathrm{M}]+\mathrm{k}_{\mathrm{o}}[\mathrm{D}]+\mathrm{k}_{\mathrm{o}}{ }^{\prime}[\mathrm{A}]^{0.5}}
$$

Assuming that $\mathrm{k}_{\mathrm{i}}[\mathrm{M}]>>\mathrm{k}_{\mathrm{o}}[\mathrm{D}]+\mathrm{k}_{\mathrm{o}}^{\prime}[\mathrm{A}]$,

$$
\mathrm{R}_{\mathrm{p}}=\mathrm{k}_{\mathrm{p}} \mathrm{k}_{\mathrm{t}}^{-0.5}\left(2 \mathrm{k}_{\mathrm{d}}\right)^{0.5} \mathrm{~K}^{0.5} \mathrm{M}[\mathrm{D}]^{0.5}[\mathrm{~A}]^{0.5} .
$$

The experimental results for MBA polymerization indicate that the mechanism just described may be operative. The initiation proposed was of radical nature, and the decomposition of the charge-transfer complex proceeded without the participation of the monomer.

\section{Conclusions}

Cyclopolymerization kinetics of $\mathrm{N}_{\mathrm{N}} \mathrm{N}^{\prime}$-methylenebisacrylamide monomer has been investigated exclusively using charge-transfer complex. Kinetic parameters are obtained from plots of the rate of polymerization versus monomer concentration and from plots of the rate of polymerization versus the square root of the concentration of the initiators. These results will serve as a new process to synthesis many high-performance polymers.

\section{References}

Butler, G.B., Angel, R.J. (1957). Preparation and polymerization of unsaturated quaternary ammonium compounds VIII. A proposed alternating intramolecular chain propagation. J. Am. Chem. Soc., 79, 3128.

Corfield, G. C. (1972). Cyclopolymerization, Chemical Society Reviews 1.

Gopalan, A., Venuvanalingam, P., Manickam, S.P., Venkatarao, K., Subbaratnam, N.R. (1982). Cyclopolymerization initiated by peroxodisulfate ion and metal ion catalysis. Eur.Polym.J., 18, 531.

Hatada, K., Okamoto, Y., Yuki, H. (1976). Proton spin-lattice relaxation times of polymers of various tacticities in solution. J. Polym.Sci., Polym. Edn., 14, 51-53. 
Kothandaraman, H., Arumugasamy, N. (1984b). A study on vinyl polymerization initiated by a charge transfer complex. Eur. Polym. J., 20, 1195-1197.

Kothandaraman, H., Arumugasamy, N. (1984c). Kinetics of polymerization of vinyl monomers initiated by dimethylaniline - tosyl chloride system. Indian J. Chem., sect. A, 23A, 155-156.

Kothandaraman, H., Arumugasamy, N. (1986a). Charge-transfer interaction between N,N-dimethylaniline and aromatic sulphonyl chlorides. J. Chem. Soc. Perkin. Trans.II, 1115-1116.

Michael A. Meador. (2003). Polyimides by photochemical cyclopolymerization. US patent No. 6593389.

Ohya, T., Otsu, T. (1983). Head-to-head vinyl polymers. XI. Preparation and Characterization of poly(methyl acrylate) and poly(methyl methacrylate) consisting of head-to-head and head-to-tail units through cyclopolymeerization of acrylic and methacrylic anhydrides. J.Polym.Sci., Polym.Chem.Edn., 21, 3503-3515.

Park, S., Okada, T., Takeuchi, K., Osakada, K. (2010). Cyclopolymerization of Monoterminal 1,6-Dienes Catalyzed by Pd Complexes. Macromolecules., 43, 7998-8006.

Park, S., Takeuchi, K., Osakada, K. (2006). Pd Complex-Promoted Cyclopolymerization of Functionalized $\alpha, \omega$-Dienes and Copolymerization with Ethylene to Afford Polymers with Cyclic Repeating Units. J.Am.Chem. Soc., 128, 3510-3511.

Parthsarathy, T., Uma, V. (2004). Dye-sensitized photopolymerization of N,N'-methylenebisacrylamide by initiation with eosin-ascorbic acid system., J. Chem. Sci., 116, 115-118.

Ratnasabapathy, S., Marisami, N., Manickam, S.P., Venkatrao, K., Subbaratnam, N.R. (1988). Kinetics of Cyclopolymerization of N,N'-Methylenebisacrylamide Initiated by Redox Couples with Mn(III). Part III. $J$. Macromol. Sci. Chem., A25, 943.

Satis, C.S., Satish, K.P., Shivakumar, H.G. (2006). Hydrogels as controlled drug delivery systems: Synthesis, crosslinking, water and drug transport mechanism. Indian Journal of Pharmaceuticals., 68,133-140.

Subbaratnam, N.R., Manickam, S.P., Venuvanalingam, P., Gopalan, A. (1986). Cyclopolymerization Initiated by Peroxydisulfate Ion and Metal Ion Catalysis. J. Macromo. Sci. Chem., A23, 117-128.

Timy P. Jose, Sharanappa T. Nandibewoor, Suresh M. Tuwar. (2006). Kinetics and Mechanism of the Oxidation of Vanillin by Hexacyanoferrate(III) in Aqueous Alkaline Medium. J. Soln. Chem., Polym. Chem. Edn., 21, 3503-3515.

Umayavalli, M., Gopalakrishnan, N., Ratnasabapathy, S., Manickam, S.P. (1995). Cyclopolymerization of divinyl monomer with manganese (VII) - thiol redox pair. Poly. Int., 38, 363-366.

Table 1. Job's method of continuous variation

\begin{tabular}{|c|c|c|}
\hline \multicolumn{3}{|c|}{$[\mathrm{DMA}]+[\mathrm{Bscl}]=0.2 \mathrm{~mol} \cdot \mathrm{L}^{-1}$} \\
\hline Mole percentage of DMA & Mole percentage of the acceptors & $\begin{array}{c}\text { O.D. } \lambda_{\max } \\
\text { DMA-Bscl } 336 \mathrm{~nm}\end{array}$ \\
\hline 10 & 90 & 0.42 \\
20 & 80 & 0.68 \\
30 & 70 & 0.83 \\
50 & 50 & 0.95 \\
70 & 30 & 0.73 \\
80 & 20 & 0.52 \\
90 & 10 & 0.26 \\
\hline
\end{tabular}


Table 3. Dependence of $\mathrm{R}_{\mathrm{p}}$ on [MBA], [DMA] and [Bscl]

\begin{tabular}{|c|c|c|c|c|c|c|c|c|c|c|c|c|c|}
\hline \multicolumn{4}{|c|}{$\begin{array}{c}\text { Effect of [MBA] } \\
\text { [Bscl\}:9.1 } \\
\text { [DMA]:1.33 }\end{array}$} & \multicolumn{5}{|c|}{$\begin{array}{c}\text { Effect of [DMA] } \\
\text { [MBA]:4.9 } \\
\text { [Bscl]:9.1 }\end{array}$} & \multicolumn{5}{|c|}{$\begin{array}{c}\text { Effect of [Bscl] } \\
\text { [MBA]:3.87 } \\
\text { [DMA]:3.33 }\end{array}$} \\
\hline [MBA] & $\mathbf{R}_{\mathbf{p}}$ & $3+\log R_{p}$ & $6+\log R_{p}$ & [DMA] & $\mathbf{R}_{\mathrm{p}}$ & $3+\log [\mathrm{DMA}]$ & $6+\log R_{p}$ & {$[\text { DMA] }]^{0.5}$} & [Bscl] & $\mathbf{R}_{\mathbf{p}}$ & $3+\log R_{p}$ & $6+\log R_{p}$ & {$[\mathrm{Bscl}]^{0.5}$} \\
\hline 2.94 & 1.61 & 1.4692 & 1.2068 & 0.67 & 2.4 & 0.826 & 1.3802 & 8.16 & 4.55 & 1.6 & 1.6580 & 1.2041 & 21.3 \\
\hline 3.92 & 2.3 & 1.5942 & 1.3617 & 1.33 & 3.1 & 1.1238 & 1.4914 & 11.5 & 9.13 & 2.4 & 1.9604 & 1.3802 & 30.2 \\
\hline 5.89 & 3.62 & 1.7702 & 1.5587 & 2.0 & 4.12 & 1.301 & 1.6149 & 14.1 & 18.2 & 18.2 & 2.2601 & 1.5051 & 42.7 \\
\hline 7.85 & 4.13 & 1.8952 & 1.616 & 2.67 & 4.78 & 1.4265 & 1.6794 & 16.3 & 27.4 & 27.4 & 2.4378 & 1.6021 & 52.2 \\
\hline 9.82 & 5.52 & 1.9921 & 1.8952 & 3.33 & 5.6 & 1.5228 & 1.7482 & 18.2 & 36.5 & 36.5 & 2.5623 & 1.6684 & 60.3 \\
\hline
\end{tabular}

All concentrations are expressed in $10^{-2} \mathrm{~mol} \mathrm{litre}^{-1}$;

$R_{p}$ values in $10^{-5} \mathrm{molLs}^{-1}$;

$[\mathrm{Bscl}]^{0.5}$ values in $10^{-2}\left(\mathrm{moll}^{-1}\right)^{0.5}$;

$[\mathrm{DMA}]^{0.5}$ values in $10^{-2}\left(\mathrm{moll}^{-1}\right)^{0.5}$.

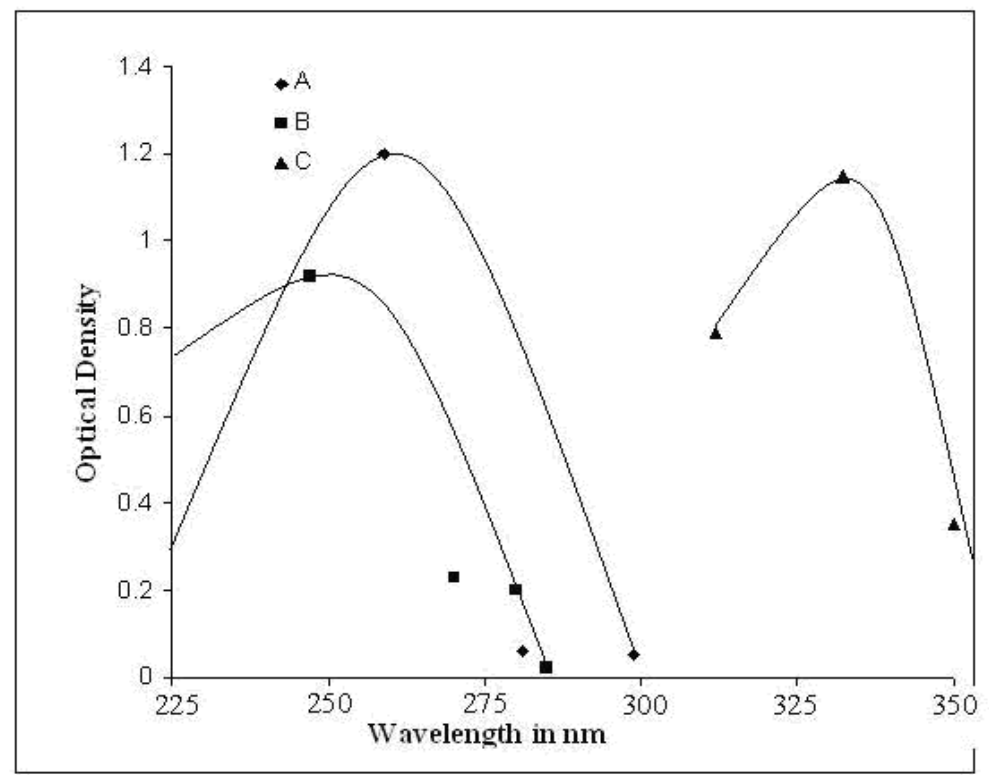

Figure 1. UV-Spectra of DMA and Bscl with spectra of DMA-Bscl complex. A. DMA; B. Bscl; C. Mixture containing DMA and Bscl 


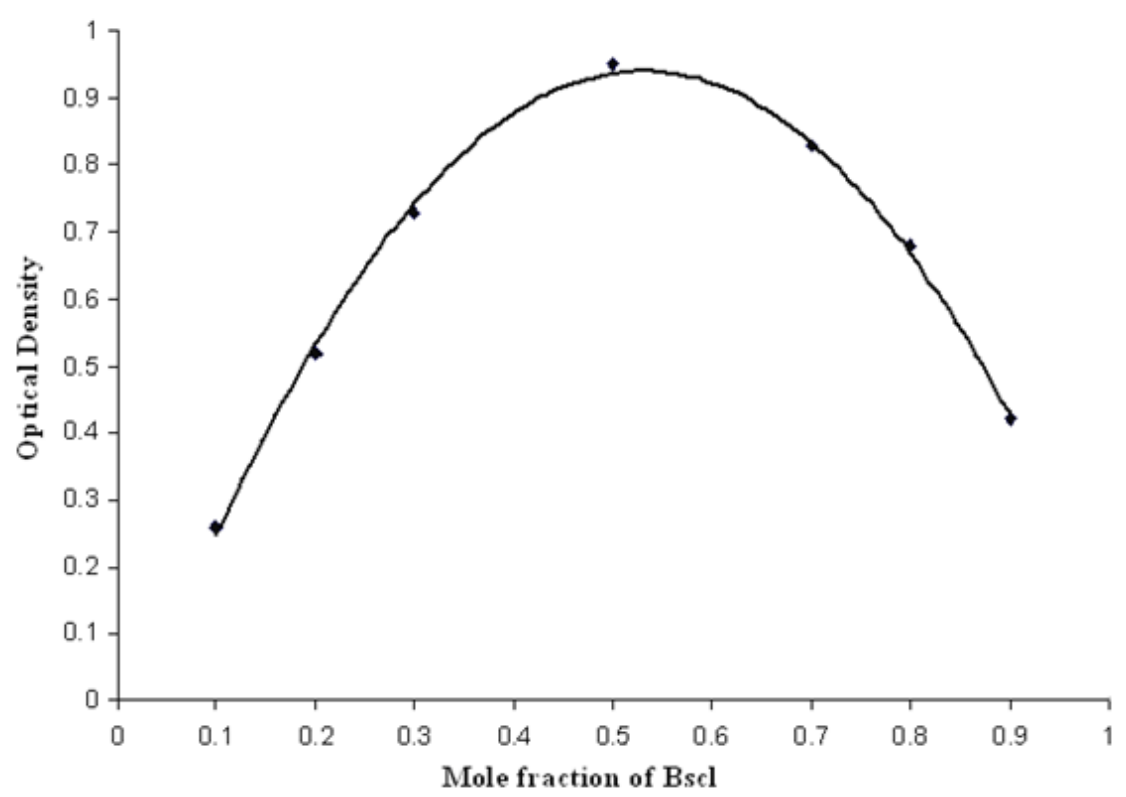

Figure 2. Jobs' method of continuous variation; $[\mathrm{DMA}]+[\mathrm{Bscl}]=0.2 \mathrm{~mol} \cdot \mathrm{L}^{-2}$<smiles>CN(C)c1ccccc1</smiles><smiles>CN(c1ccccc1)C(Cl)(Oc1ccccc1)c1ccccc1</smiles><smiles>C[N+](C)(c1ccccc1)c1ccccc1</smiles><smiles></smiles><smiles>CN(C)c1ccccc1</smiles>

(R)

Figure 3. Formation of CT comples and its thermal decomposition 


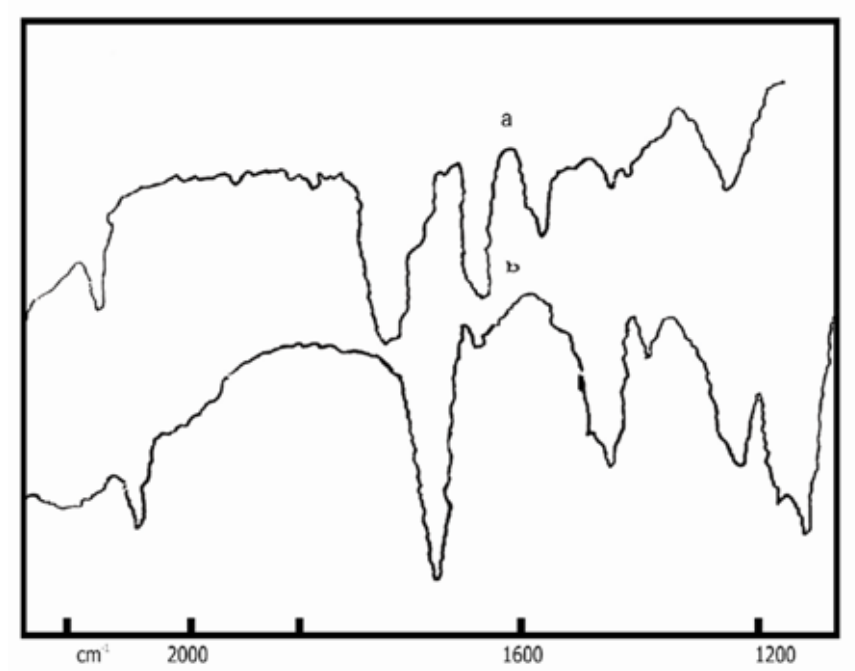

Figure 4. IR Spectra (a) Poly MBA (b) MBA

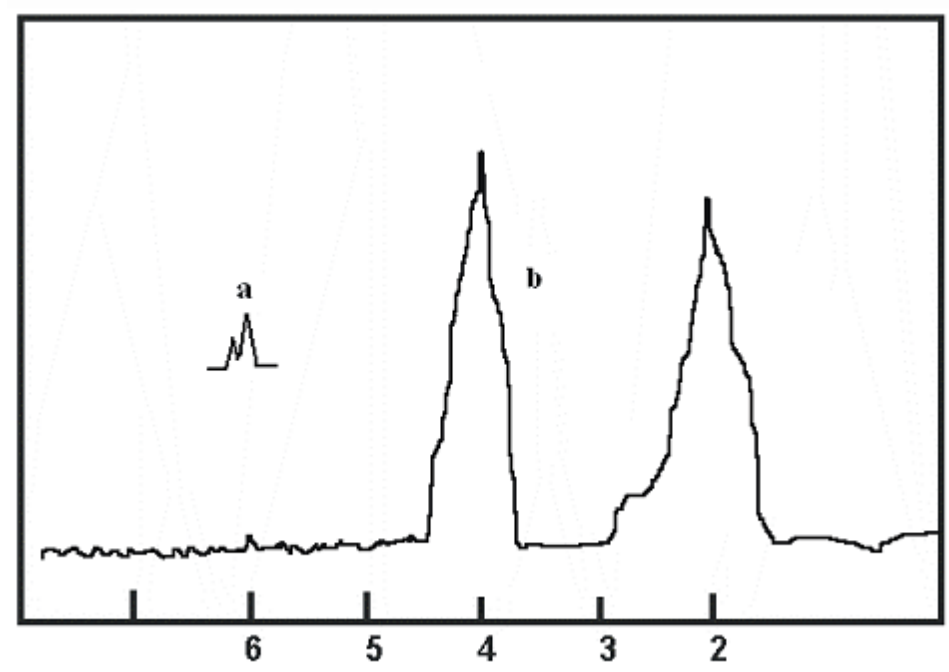

Figure 5. HNMR Spectra (b) MBA (a) $\mathrm{CH}_{2}=\mathrm{CH}_{2}$ Protons in MBA Recorded in $\mathrm{CDCl}_{3}$ at $100{ }^{\circ} \mathrm{C}$ 


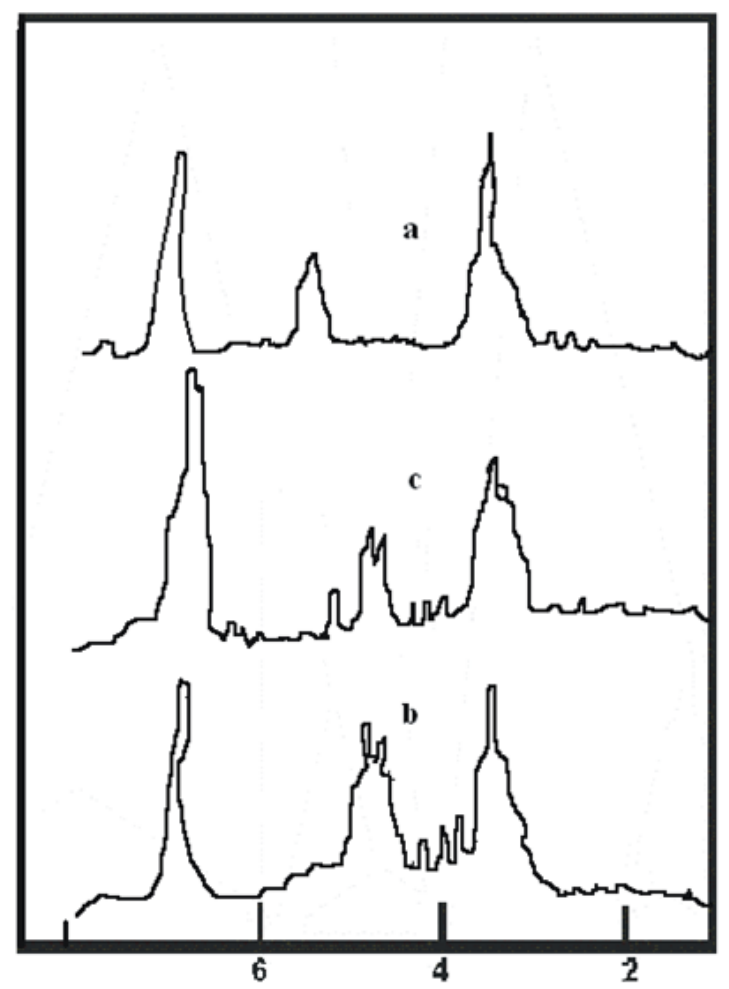

Figure 6. HNMR Spectra Model Compounds (a) for Pure HH (b) for Pure HT and (c) 30/70 HH/HT addition Propagation

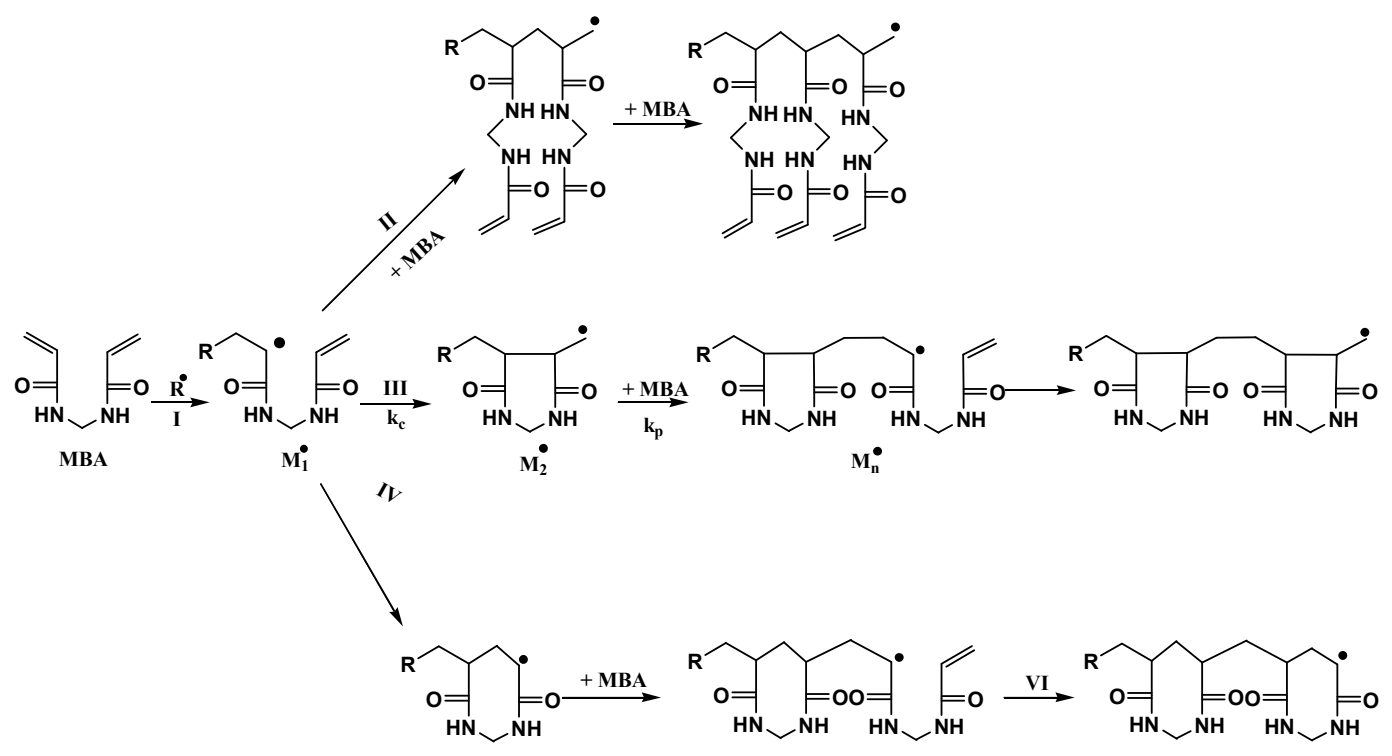

Figure 7. Propagation Sequences

Step I: Initiation by free radical generated by the decomposition of redox pair complex

Step II: Vinyl propagation by intermolecular HT addition

Step III: Cyclopolymerization by HH intramolecular addition giving seven membered ring unit

Step IV: Cyclopolymerization by HT intra and intermolecular addition propagation giving eight membered ring unit 


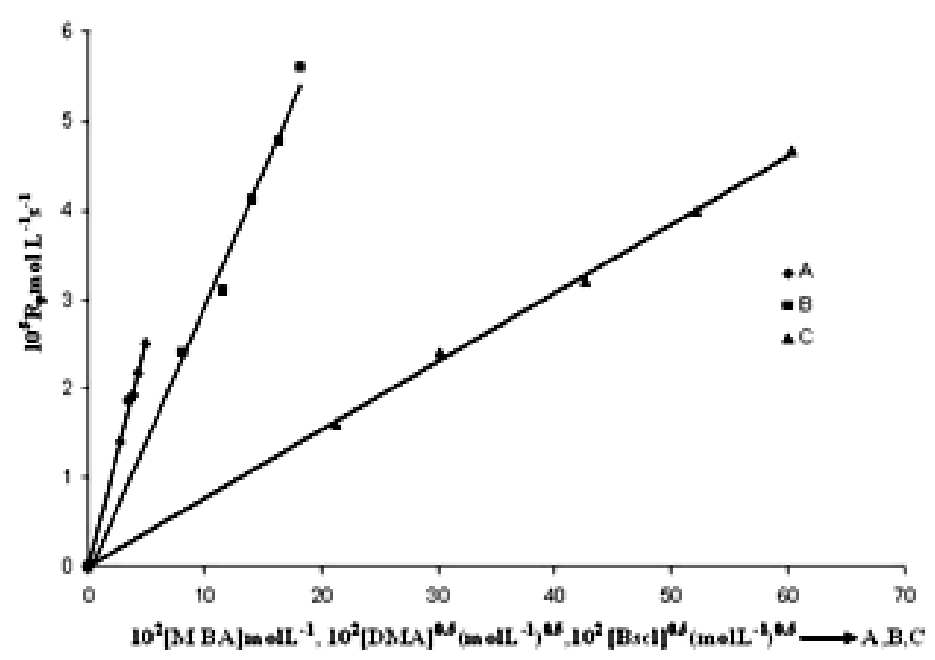

Figure 8. (A) Dependance of $\mathrm{R}_{\mathrm{p}}$ on $[\mathrm{MBA}]$; [Bscl] $=18 \times 10^{-2}$, [DMA] $=1.33 \times 10^{-2}$

(B) Dependance of $\mathrm{R}_{\mathrm{p}}$ on $[\mathrm{DMA}]$; [Bscl] $=9.1 \times 10^{-2}$, $[\mathrm{MBA}]=4.9 \times 10^{-2}$

(C) Dependance of $\mathrm{R}_{\mathrm{p}}$ on $[\mathrm{Bscl}]$; [Bscl] $=3.33 \times 10^{-2}$, [MBA] $=3.87 \times 10^{-2}$

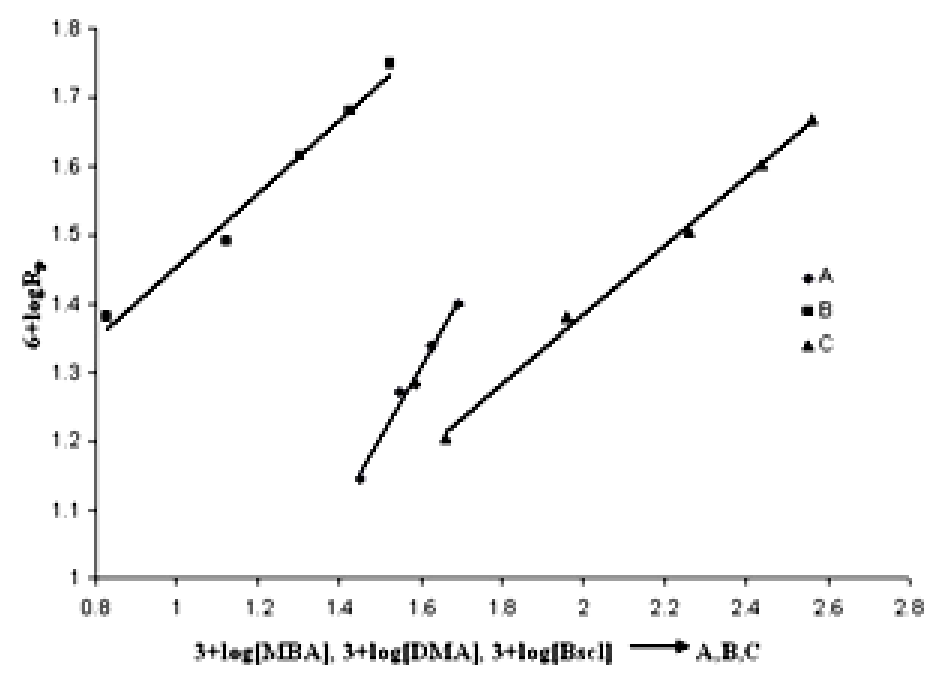

Figure 9. (A) Dependance of $\log \mathrm{R}_{\mathrm{p}}$ on $\log [\mathrm{MBA}]$; $[\mathrm{Bscl}]=18 \times 10^{-2}$, [DMA $]=1.33 \times 10^{-2}$

(B) Dependance of $\log \mathrm{R}_{\mathrm{p}}$ on $\log [\mathrm{DMA}]$; $[\mathrm{Bscl}]=9.1 \times 10^{-2},[\mathrm{MBA}]=4.9 \times 10^{-2}$

(C) Dependance of $\log \mathrm{R}_{\mathrm{p}}$ on $\log [\mathrm{Bscl}]$; $[\mathrm{Bscl}]=3.33 \times 10^{-2}$, [MBA] $=3.87 \times 10^{-2}$ 